\title{
Surgical Management of Parapharyngeal Space Tumors: Our Experience
}

\author{
Rajendra B. Metgudmath • Anjali R. Metgudmath • \\ Prakash R. Malur • Vinita V. Metgudmath • \\ Amal T. Das
}

Received: 11 December 2011/ Accepted: 3 February 2012/Published online: 15 February 2012

(C) Association of Otolaryngologists of India 2012

\begin{abstract}
The purpose of this study is to evaluate parapharyngeal space (PPS) tumors with regards to clinicopathological features and pre-operative assessment and also to analyze the benefits of transcervical approach in the management of PPS tumors. We performed retrospective analysis of patients who had undergone transcervical resection of PPS tumors from May 2006 to May 2009 at KLES Dr. Prabhakar Kore Hospital and M. R. C, Belgaum. Patients were diagnosed on the basis of clinical examination, FNAC and magnetic resonance imaging. All patients were observed with an annual follow-up of at least 3 years by physical examination and ultrasonographic evaluation. The total number of patients were four; with three males and one female patient. Their age varied from 20 to 55 years with a mean age of 36 years. There were two cases of neurilemomas, one case of carotid body tumor and one case of pleomorphic adenoma. All four patients underwent surgical excision of tumor by transcervical approach. There were no major post-operative complications. All four patients are alive with no recurrence in the 3 year follow-up period. Transcervical approach is
\end{abstract}

R. B. Metgudmath $(\bowtie) \cdot$ V. V. Metgudmath · A. T. Das Department of Otorhinolaryngology, Head and Neck Surgery, Jawaharlal Nehru Medical College, KLE University, 95/C, Anugraha, Shanti-nagar, M. G. Road, Tilakwadi, Belgaum 590006, Karnataka, India

e-mail: drrajmet@yahoo.co.in

A. R. Metgudmath

Department of General Medicine, Jawaharlal Nehru Medical

College, KLE University, Belgaum, Karnataka, India

P. R. Malur

Incharge Histo-pathologist, K. L. E. S. Dr. Prabhakar Kore Hospital and M. R. C, KLE University, Belgaum, Karnataka, India versatile, flexible and provides good access to the narrow PPS. This approach also provides good vascular control and reduced risk of post-op complications. We recommend transcervical approach even for large sized pre-styloid and post-styloid compartment benign tumors which are free from deep lobe of parotid.

Keywords Parapharyngeal space tumors .

Pleomorphic adenoma $\cdot$ Neurilemoma .

Transcervical approach

\section{Introduction}

The parapharyngeal space (PPS) is a deep potential neck space often described as an inverted pyramid that extends from the base of skull to the greater cornu of the hyoid bone. It is divided into pre-styloid and post-styloid compartment on the basis of its relationship to the tensor-vascular-styloid fascia. Tumors arising from the PPS are rare and account for only $0.5 \%$ of head and neck neoplasms [1,2]. Wide variety of histologic types has been described of which $80 \%$ are benign and $20 \%$ are malignant [1]. The most frequent primary tumor of PPS is salivary gland neoplasms followed by neurogenic tumors like neurilemomas or paragangliomas [1]. The complex anatomy of the parapharyngeal region makes the clinical examination of this area difficult and an unreliable method for the assessment of these tumors. Therefore imaging modalities like computer tomography (CT) and magnetic resonance imaging (MRI) are vital to determine the extent of the lesion, to delineate its relationship to carotid artery, to assess its resectability, to rule out any intracranial involvement and guide the operating surgeon in planning the right surgical approach. Fine needle aspiration cytology (FNAC) is mandatory to avoid any 
histologic surprise and should be performed following diagnostic imaging in order to exclude vascular lesion.

The purpose of this article is to review our experience with PPS tumors and to recommend transcervical approach without mandibulotomy in the management of even large sized pre and post-styloid space tumors.

\section{Materials and Method}

We conducted a retrospective review of four patients who underwent elective excision of PPS tumors between May 2006 to May 2009 at KLES Dr. Prabhakar Kore Hospital and Medical Research Centre, Belgaum. The clinical features, histopathology and radiographic features of the tumor, surgical management and outcome were analysed. Depending on the location of tumour in PPS, the cases were grouped into pre-styloid space tumors or post-styloid space tumors. All four patients underwent transcervical excision of the tumor with horizontal incision taken at the level of hyoid bone. The submandibular gland was retracted/removed to gain access to the parapharynx. Access to the apex of pre-styloid space tumors was gained between the digastric muscle and the mandible whereas access to the post-styloid space tumors was obtained medial to posterior belly of digastric. The digastric tendon was divided when additional access to the PPS was required. Tumors were removed without damaging the capsule or/and the nerve of origin. All patients were observed with an annual follow-up of at least 3 years by physical examination and ultrasonographic evaluation of neck.

\section{Results}

The total number of patients in this study were four; with three males and one female patient. Their age varied from 20 to 55 years with a mean age of 36 years. The first three patients presented with symptoms of neck swelling, difficulty in swallowing and change in voice. In all three cases the tonsil, soft-palate and lateral oropharyngeal wall were pushed medially with firm and non-pulsatile swelling in the submandibular region. Our fourth patient presented with a painless slow growing swelling over the right side of the neck.

All the four patients were subjected for MRI of neck with gadolinium contrast. In three patients the tumor was located in post-styloid compartment while in one patient the tumor was situated in pre-styloid compartment (Table 1). In our first and third patient with post-styloid space tumors, MRI revealed a well-defined heterogeneous mass, the largest measuring $9.0 \times 7.0 \times 4.7 \mathrm{~cm}$ with predominantly hyper-intense on $\mathrm{T} 2$ weighted images and iso-intense on T1 weighted images (Fig. 1). These features were suggestive of neurogenic tumor. In our fourth case with post-styloid compartment tumor, MRI revealed a large well-defined ovoid intensely enhancing mass measuring $4.8 \times 3.5 \times 3.2 \mathrm{~cm}$ epicentered over the right carotid bifurcation insinuating between the internal and external carotid arteries causing splaying of the carotid bifurcation (Lyre sign positive as seen in Fig. 2). These features were suggestive of carotid body tumor. MRI of the pre-styloid compartment tumor revealed a well-circumscribed mass measuring $7.0 \times 6.1 \times 4.2 \mathrm{~cm}$ with homogeneous enhancement after gadolinium administration, suggestive of pleomorphic adenoma. No adjacent bony erosion was seen in any of the four cases. Tissue diagnosis was established prior to surgery by performing fine needle aspiration of the tumor except in the case of intensely enhancing poststyloid vascular tumor.

All four patients underwent surgical excision of tumor through transcervical approach (Fig. 3). Microscopic examination of two of the excised neurogenic tumors from post-styloid compartment showed Antoni A regions made of spindle shaped cells in wavy bundles with prominent palisades and Verocay bodies interspersed with Antoni B regions suggestive of neurilemomas. The histology of the vascular post-styloid compartment tumor revealed nests of cuboidal cells separated by highly vascular fibrous stroma suggestive of carotid body tumor. The histology of the prestyloid compartment tumor showed myxomatous stroma in which there were spindle shaped myoepithelial cells, epithelial cells and glandular elements with foci of pseudo cartilage suggestive of pleomorphic adenoma (Fig. 4). Postoperative period was uneventful except for patient with the large $9.0 \mathrm{~cm}$ tumor, who had paresis of the left vocal cord post-operatively which recovered completely following speech therapy and was started on oral feeds. All the four patients are alive with no recurrence in the 3 year follow-up period.

\section{Discussion}

Approximately $50 \%$ of tumours of the PPS are salivary gland in origin and 20\% are neurogenic [1]. Tumors of the pre-styloid region are generally limited to salivary gland lesions, lipomas and very rarely neurogenic tumors [2, 3]. Neurogenic tumors occur most commonly in the post-styloid space with majority arising from the vagus nerve.

Parapharyngeal space tumors generally are slow growing and present as asymptomatic masses. With time they can cause obstructive symptoms, as seen in our first case along with temporary or permanent loss of nerve function. Pain is uncommon. They cause medial displacement of 
Table 1 Summary of patients in the study

\begin{tabular}{lllllll}
\hline No. & Age/Sex & Histo-pathology & Site & Size $(\mathrm{cm})$ & Approach & Outcome at 3 years follow-up \\
\hline 1 & 20/M & Neurilemoma & Post-styloid & $9.0 \times 7.0 \times 4.7$ & Transcervical & Alive, NR \\
2 & $55 / \mathrm{M}$ & Pleomorphic adenoma & Pre-styloid & $7.0 \times 6.1 \times 4.2$ & Transcervical & Alive, NR \\
3 & $27 / \mathrm{F}$ & Neurilemoma & Post-styloid & $6.7 \times 4.4 \times 4.6$ & Transcervical & Alive, NR \\
4 & $42 / \mathrm{M}$ & Carotid body tumor & Post-styloid & $4.8 \times 3.5 \times 3.2$ & Transcervical & Alive, NR \\
\hline
\end{tabular}

$N R$ No recurrence
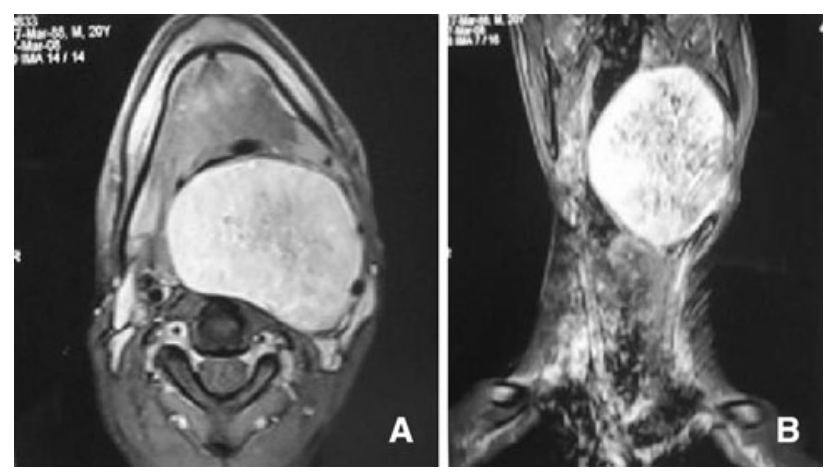

Fig. 1 MRI scan shows large post-styloid space neurilemoma measuring $9.0 \times 7.0 \times 4.7 \mathrm{~cm}$ shown in a axial section and in b coronal section
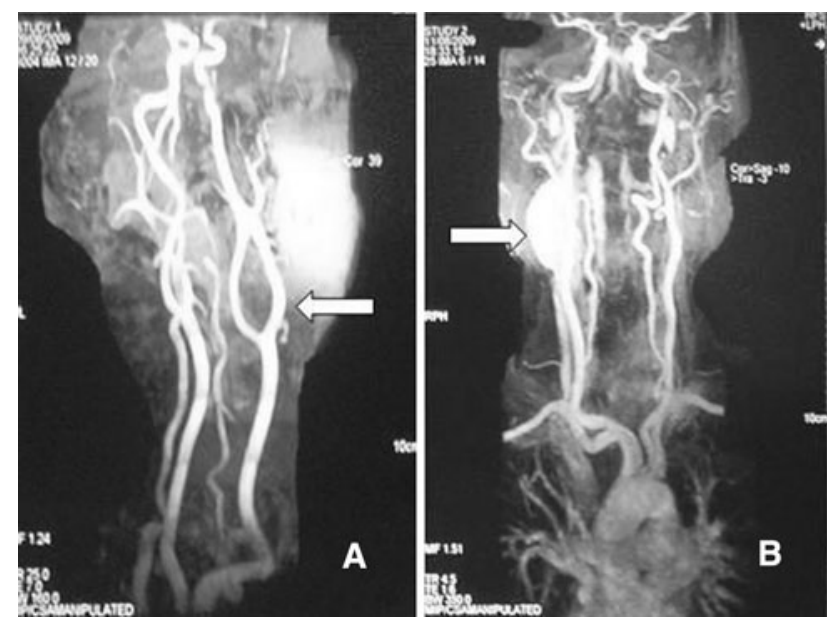

Fig. 2 a Digital subtraction angiography showing splaying of internal and external carotid arteries (Lyre sign). b MR angiography showing the relation of the ovoid carotid body tumor at the carotid artery bifurcation

lateral oropharyngeal wall [3, 4]. Tumours of the PPS are often misdiagnosed as infections or tumors of the tonsil. Signs suggestive of malignant transformation include facial nerve deficit, changes in consistency, rapid increase in size and recent onset of pain.

Radiographic study of all PPS tumors is essential. MRI with gadolinium is the examination of choice $[4,5]$. It helps to define soft tissue characteristics of the neoplasm accurately and gives important information regarding

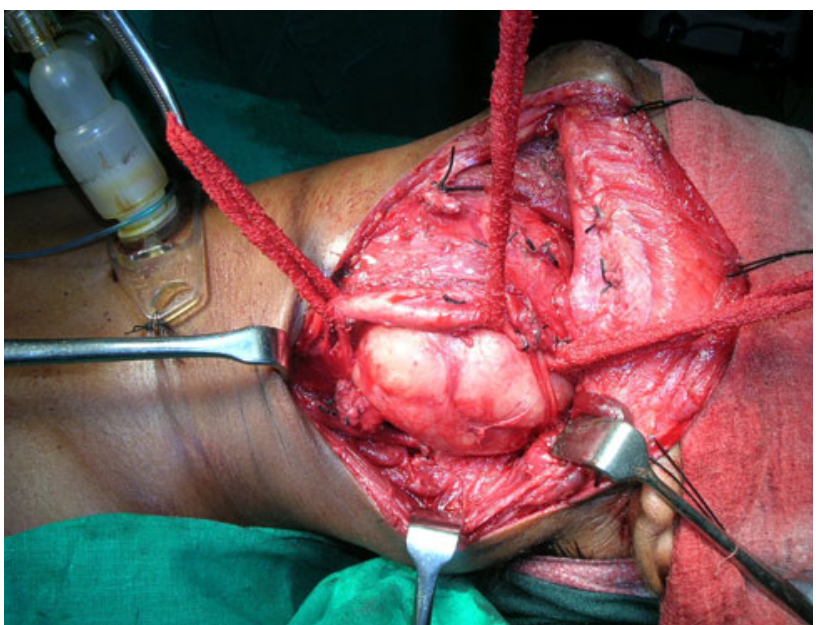

Fig. 3 Tumour exposure by transcervical approach

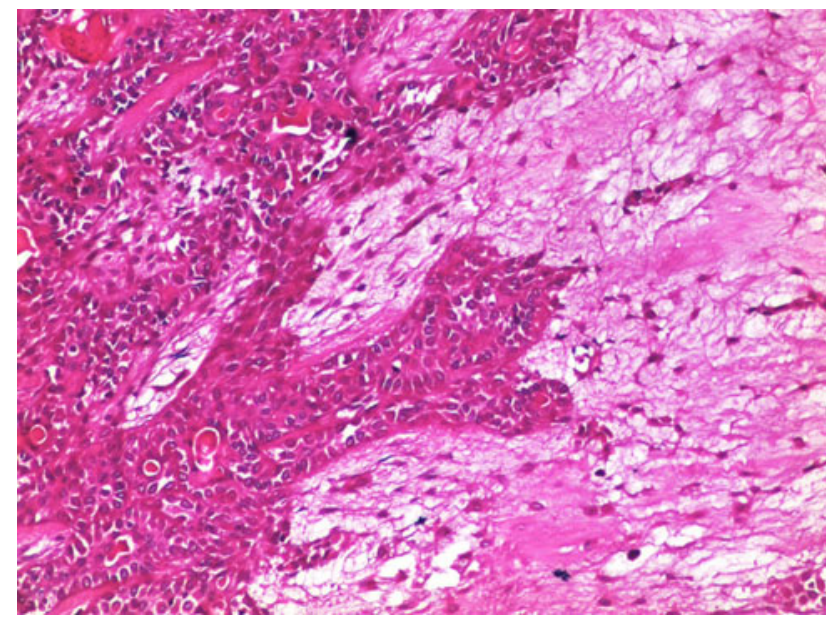

Fig. 4 Pleomorphic adenoma: presence of myoepithelial cells, epithelial cells and glandular elements scattered in myxomatous stroma. $\mathrm{H}$ and $\mathrm{E}, 10 \mathrm{x}$

anatomical location, local invasion, regional metastasis and tumor type [6]. A CT with contrast would enable to add valuable information regarding the tumor relation to bony structures, presence of calcifications and its relationship to great vessels. Besides it is used as an adjunct to exclude mandibular infiltration [6]. Angiography is recommended for all enhancing lesions or vascularised masses, especially 
if imaging revealed splaying of the carotid bifurcation (Lyre sign).

Fine needle aspiration cytology will provide useful information to the operating surgeon and help in choosing the right surgical approach. Under no circumstance should biopsy of the PPS mass be performed prior to obtaining imaging studies so as to exclude vascular lesions. In our three cases, the FNAC report was correct in all instances. Open neck or trans-oral biopsies is contraindicated for most PPS tumors since opening the tumor capsule increases the risk of recurrence [1].

If paragangliomas are suspected, $24 \mathrm{~h}$ urine collection of catecholamines including vanillyl mandelic acid (VMA) and metanephrines should be carried out. If the catecholamines are positive, then do a meta iodinated benzyl guanidine (MIBG) scan to trace catecholamine uptake and storage. The possibility of multicentric paragangliomas have to be ruled out by performing MRI, CT and if indicated bilateral carotid angiography. CT evaluation of the adrenal glands should always be done in multiple paraganglioma syndromes.

Several surgical approaches such as transoral, transcervical (with or without mandibulotomy), transparotid, transmandibular and infra temporal fossa, combined transoral-external approach, cervical transpharyngeal with midline mandibulotomy have been described in the management of PPS tumors [1]. The selection of surgical approach is dictated by the size of tumor, its location, relation to major vessels and the index of suspicion of malignancy. As majority of these tumors are benign, the approach which offers minimum surgical morbidity must be selected [3].

Transcervical approach provides adequate exposure for excision of majority of PPS neoplasms. This approach has been recommended by many authors as the best access route for removal of PPS tumors. Bass described the removal of submandibular gland with identification of the marginal mandibular branch of facial nerve. Though it has been commonly used for post-styloid masses; even prestyloid compartment tumors free from the deep lobe of parotid can be easily excised with this approach, as performed in our case.

Shamblin classification of carotid body tumors was used to predict the risk of internal carotid artery compromise [7]. The surgical excision with careful sub-adventitial dissection is the treatment of choice for most carotid body paragangliomas (Shamblin I and II). The Shamblin III type requires resection of the external and/or internal carotid artery [7]. In our case, the carotid body tumor was of Shamblin type I and was excised by transcervical approach.

Transparotid approach is commonly used for deep lobe parotid tumors, especially for dumbbell shaped lesions and was initially described by Bass [8].
Transoral approach offers a direct route to tumors presenting in the oropharynx. Major disadvantage to this approach are incomplete exposure and provides no proximal control of the great vessels, hemorrhage, increased risk of infection and increased risk of damage to the facial nerve. Several authors have condemned the transoral approach as inadequate and unsafe [3]. Authors have stated that the transoral approach should be used only for small lesions not extending to the styloid process [8]. Betka et al. performed transoral resection of tumor in 23 patients without any complications based on which they claim that transoral approach is a safe surgery [9].

Cervical transpharyngeal approach was first described by Roux in 1981. This approach has been used by several surgeons for the removal of large malignant or highly vascular tumors in the PPS [3]. This approach can be combined with a lower lip incision to gain access to the pharynx via a mandibulotomy.

The infratemporal fossa approach was described by Fisch for PPS tumors involving the skull base or extending into the infratemporal fossa [10]. This approach gives direct surgical access to the entire length of the lateral skull base, from the nasopharynx and PPS anteriorly to the foramen jugular posteriorly [10].

Transmandibular approach was advocated by DeCampora in 1984. The mandibulotomy may be required for vascular lesions in the upper part of the PPS or for malignant neoplasms, where good access and optimal vessel control is vital [3]. Disadvantages include an extensive external scar, risk of complications associated with healing of the bone and teeth, contamination of the neck wound and the need for tracheotomy [3]. Even with this elaborate exposure, the tumor must be bluntly separated from the base of the skull and neurovascular structures because of limited visibility.

Although the best exposure of the PPS is gained by a mandible splitting procedure either a lateral or midline mandibulotomy, we do not feel that the morbidity of these procedures justify their use in benign tumors. Giant tumors even up to $11 \times 9 \times 9 \mathrm{~cm}$ have been excised without splitting the mandible. These more radical approaches may be indicated when malignancy is suspected [1].

Complications of surgery of the PPS include tumor recurrence, nerve injury, vessel injury, hematoma and osteotomy site complications [1]. Tumor recurrence is possible, especially if the capsule is broken and tumor spillage occurs [3].

Post-operative radiotherapy is recommended for malignant tumors, unresectable paragangliomas and patients who represent a poor surgical risk [3]. These include patients with internal carotid artery involvement who fail balloon occlusion or patients with contralateral cranial nerve deficits in whom resection would lead to significant reduction 
in the quality of life. Most surgeons for malignant tumors recommend radiation therapy as an adjunct to surgery in patients with high-grade malignancies, inoperable tumors and when adequate oncologic resection margin cannot be obtained.

\section{Conclusion}

The surgeon must be well versed in the complex anatomy of this area to plan for safe removal of the tumor. Transcervical approach is versatile, flexible and provides good access to the narrow PPS. This approach also provides good vascular control and reduced risk of post-op complications. We recommend transcervical approach even for large sized pre-styloid and post-styloid compartment benign tumors which are free from deep lobe of parotid.

Conflict of interest The authors have no conflict of interest.

\section{References}

1. Olsen KD (1994) Tumors and surgery of the parapharyngeal space. Laryngoscope 104(Suppl 63):1-28
2. Batsakis JG, Sneige N (1989) Parapharyngeal and retropharyngeal space diseases. Ann Otol Rhinol Laryngol 98:301-303

3. Carrau RL, Myers EN, Johnson JT (1990) Management of tumors arising from the parapharyngeal space. Laryngoscope 100:583-589

4. Allison RS, van der Waal I, Snow GB (1989) Parapharyngeal tumors: a review of 23 cases. Clin Otolaryngol 14:199-203

5. Shahab R, Heliwell T, Jones AS (2005) How we do it: a series of 114 primary parapharyngeal space neoplasms. Clin Otolaryngol 30:364-383

6. Stambuk HE, Patel SG (2008) Imaging of the parapharyngeal space. Otolaryngol Clin North Am 41:77-101

7. Kohler HF, Carvalho AL, Mattos Granja NV, Nishinari K, Kowalski LP (2004) Surgical treatment of paragangliomas of the carotid bifurcation: results of 36 patients. Head Neck 26(12): 1058-1063

8. Bass RM (1982) Approaches to the diagnosis and treatment of tumors of the parapharyngeal space. Head Neck Surg 4:281-289

9. Betka J, Chovanec M, Klozar J, Taudy M, Plzak J, Kodetova D, Lisy J (2010) Transoral and combined transoral-transcervical approach in the surgery of parapharyngeal tumors. Eur Arch Otorhinolaryngol 267:765-772

10. Shahinian H, Dirnier C, Fisch U (1995) Parapharyngeal space tumors: the infratemporal fossa approach. Skull Base Surg 5(2): $73-81$ 